\title{
EL USO DE LA TECNOLOGÍA EN ELAULA DE MATEMÁTICAS: DIFERENCIAS DE GÉNERO DESDE LA PERSPECTIVA DE LOS DOCENTES
}

\author{
Ursini, Sonia ${ }^{1}$; Sanchez, Gabriel ${ }^{2}$; Orendain, Mónica ${ }^{3}$ y Butto, Cristianne ${ }^{1}$ \\ ${ }^{1}$ Departamento de Matemática Educativa. CINVESTAV. Av. Politécnico Nacional. 2502 México D.F. \\ ${ }^{2}$ Facultad de Psicología. FES Zaragoza. UNAM. México DF \\ ${ }^{3}$ Dirección General de Materiales y Métodos Educativos. SEP. México DF \\ soniaul2002@yahoo.com.mx \\ josegsr@servidor.unam.mx \\ morendai@ilce.edu.com \\ cristianne_butto@hotmail com
}

\begin{abstract}
Resumen. Desde 1997 la Secretaría de Educación Pública en México ha promovido el proyecto nacional EMAT (Enseñanza de las Matemáticas con Tecnología) usando computadoras y calculadoras TI-92 para apoyar la enseñanza de las matemáticas en la escuela secundaria (alumnos entre 12 y 15 años de edad). En el marco de este proyecto hemos investigado como se ve afectado, según los profesores, el comportamiento de los estudiantes cuando se usa la tecnología para apoyar la enseñanza de las matemáticas. En particular se analiza si las respuestas de los profesores ponen en evidencia posibles diferencias de género. Veinticuatro docentes expresaron sus puntos de vista acerca de los cambios apreciados en 1.113 estudiantes en relación a: participación; capacidad para analizar un problema e interpretar las hojas de trabajo; iniciativa; solicitud de ayuda; dedicación; defensa de sus ideas; creatividad; preferencia por trabajo en equipo o individual. Los resultados muestran que, desde la perspectiva de los docentes, el uso de la tecnología en la clase de matemáticas modifica la mayoría de estos aspectos y estos cambios son distintos en varones y en mujeres. Además, los datos sugieren que los cambios de conducta conllevan una mayor equidad de género.
\end{abstract}

Palabras clave. Matemáticas, tecnología en la enseñanza, diferencias de género, cambios conductuales.

Summary. Since 1997 the Ministry of Education in Mexico has been sponsoring the national project EMAT (Teaching Mathematics with Technology) in which computers and TI-92 calculators are used to support the teaching of mathematics at secondary school level (children aged 12 to 15). One of our concerns during this project was to investigate if teachers consider that using technology to support the teaching of mathematics affects students' behavior. In particular, teachers' responses were analyzed to detect eventual gender differences. Twenty-four teachers gave their points of view concerning the behavioral changes of 1113 students in relation to: participation; capability to analyze a problem and to interpret the worksheets; initiative; requirement for help; dedication; defense of their own ideas; creativity; preference for working in teams or individually. The results obtained show that, from the teachers' perspective the use of technology in the mathematics classroom modifies the majority of these aspects and its impact is different for girls and boys. Moreover the behavioral changes observed seem to lead to more gender equity.

Keywords. Mathematics, technology for teaching, gender differences, behavioral changes.

\section{INTRODUCCIÓN}

En la última década ha habido una fuerte tendencia a incorporar la tecnología en el aula de matemáticas con la intención de apoyar la enseñanza y el aprendizaje de esta disciplina. Para lograr este propósito, además de equipar las aulas con la tecnología adecuada y diseñar las actividades apropiadas, es necesario conocer de qué manera la presencia de la tecnología incide en distintos aspectos que intervienen en el proceso de aprendizaje. Entre éstos, consideramos significativos algunos aspectos conductuales de los estudiantes, con los que suelen asociarse diferencias de género. Resulta, por lo tanto, importante investigar si, al introducir la tecnología en la clase de matemáticas, las diferencias de género se acentúan o se propicia una mayor equidad. Algunas cuestiones que quisiéramos responder son las siguientes: ¿Usar la tecnología en la clase de matemáticas repercute en el comportamiento de los alumnos y las alumnas dentro de la clase? Si la conducta cambia, ¿los cambios son iguales para los hombres y para las mujeres? ¿Los eventuales cambios en el comportamiento contribuyen a que las diferencias de género se acentúen o a que haya una mayor equidad de género? 
Para contestar estas preguntas se decidió recurrir, en primera instancia, a algunos profesores que llevaban entre uno y tres años usando el soporte tecnológico para enseñar matemáticas, en el marco de un proyecto piloto institucional promovido por la Secretaría de Educación Pública de México. La información así obtenida revelaría cómo perciben los docentes los cambios de comportamiento entre sus alumnos y cómo en esta percepción se reflejan sus concepciones de género. Este tipo de información resulta relevante al considerar que el concepto de género es una construcción sociocultural elaborada a partir de las diferencias sexuales. Partiendo de las diferencias biológicas, naturales, se han ido delimitando histórica y socialmente los distintos roles que hombres y mujeres tienen en las sociedades y, asociados a éstos, toda una serie de valores a los que se suelen atribuir distintas jerarquías. Como todo constructo sociocultural, también las diferencias de género se reproducen mediante la educación que tiene, entre otros, el propósito de mantener y transmitir los valores culturales que dominan en cierta sociedad. Una de las instituciones encargadas de transmitir la cultura y los valores en nuestra sociedad es la escuela y uno de sus vehículos más importantes es el profesor. La escuela, con sus normas y contenidos curriculares, junto con los profesores que se ocupan de que estas normas y contenidos se implementen, suelen ser también reproductores de las diferencias de género. Al introducir la computadora en el aula de matemáticas se modifica la cultura del salón de clase. En consecuencia, tal modificación cultural puede cambiar también el comportamiento de los estudiantes. Es importante, en consecuencia, investigar cómo perciben los profesores los cambios en el comportamiento de sus alumnos, cuando usan la tecnología en la clase de matemáticas, y cómo se reflejan en sus comentarios sus propias concepciones de género.

\section{GÉNERO, MATEMÁTICAS Y EDUCACIÓN}

Los estudios de género en el campo de la matemática educativa ya tienen una larga tradición, si bien no así en México, donde este tipo de investigaciones apenas se inicia. Durante las últimas tres décadas se ha realizado una cantidad considerable de estudios, en distintos países, para conocer cómo se relacionan las diferencias de género con el aprendizaje de las matemáticas. En particular, se ha investigado si existen diferencias de género en los logros, el desempeño y en las actitudes hacia las matemáticas, y a qué se deben (Fennema y Sherman, 1976; Hanna, 1989; Leder y Fennema, 1990; Koehler, 1990; Leder, 1992; Leder, 1996; Figueiras et al., 1998; Forgasz y Leder, 2000; Leder, 2001). También se han investigado las diferencias de género en las atribuciones causales y se ha tratado de establecer una relación entre las atribuciones causales y el logro en matemáticas (Kloosterman, 1990), así como la relación entre éste y la motivación (Middleton et al., 1990). Se ha explorado cómo se ve afectado el desempeño en matemáticas en consecuencia del trato diferenciado que dan los profesores a estudiantes varones y a estudiantes mujeres (Koehler, 1990; Kuyper y Van der Werf, 1990). Boaler (1996) investigó si los distintos enfoques que se usan para enseñar matemáticas afectan de manera distinta a alumnos y alumnas. Los resultados de su estudio indicaron que un enfoque más tradicional, que sigue un libro de texto, exacerbaba las diferencias de género, favoreciendo a los varones. Por lo contrario, un enfoque más abierto, centrado en proyectos, aminoraba estas diferencias, ya que tanto las chicas como los chicos obtenían buenos resultados. Helwig y sus colaboradores (2001), por otro lado, investigaron si el género de los estudiantes influye en cómo perciben los profesores su desempeño en matemáticas. Los resultados indicaron que, al parecer, los profesores no toman en cuenta el género de los estudiantes cuando califican su desempeño. A lo largo de varios años se señaló recurrentemente que había una tendencia a considerar las matemáticas como un dominio masculino; sin embargo, estudios recientes (Forgasz, 2001) señalan que esta actitud está cambiando y que actualmente los jóvenes ya no están viendo las matemáticas como un dominio esencialmente masculino. También se ha encontrado, de forma consistente, que existen algunas diferencias en el desempeño en matemáticas en favor de los varones, particularmente cuando se requieren altos niveles de habilidades cognitivas (Leder, 1992) o cuando las actividades requieren de habilidades geométricas, en particular de visualización (Ben-Chaim et al., 1985).

En la década pasada se ha empezado a estudiar cómo influye, en el desarrollo de las diferencias de género, la educación diferenciada que suelen recibir en la mayoría de las culturas los niños y las niñas. Subirats y Brullet (1999), por ejemplo, señalan que la presión cultural y social que se ejerce sobre el varón y la mujer promueve conductas diferenciadas que, al interiorizarse, llevan a diferentes creencias, actitudes y expectativas. Éstas, en consecuencia, pueden afectar el aprendizaje de las matemáticas (Figueiras et al., 1998). Conductas diferenciadas se pueden observar, por ejemplo, en la participación activa, en la solicitud de atención y requerimiento de ayuda en el grado en el que los alumnos y las alumnas se involucran en una actividad, en el tiempo que dedican al trabajo, en la creatividad que desarrollan y en el nivel de disciplina que se observa en su comportamiento. Investigaciones realizadas en distintos países muestran que, por lo general, los varones tienden a ser más activos en el aula y suelen participar más que las mujeres; y que tienden a solicitar más atención y ayuda que las mujeres (Meyer y Koehler, 1990; Subirats y Brullet, 1999). A este respecto, algunos investigadores señalan que una actitud activa es fundamental para la adquisición de nuevos conocimientos y no ayudar a que las mujeres la desarrollen puede ser perjudicial para su aprendizaje (Subirats y Brullet, 1999; Cooper, Marquis y Ayers-López, 1982). Sin embargo, también hacen notar que, cuando se trata de externar experiencias menos personales, como suelen ser las relacionadas con las matemáticas, la participación de las niñas aumenta considerablemente y puede llegar a rebasar la de los niños (Subirats y Brullet, 1999). Otra característica que suele resaltarse es que las mujeres son, por lo general, más dedicadas y constantes en el trabajo que los varones (Figueiras et al., 1998). Además, hay quienes afirman que, si bien no es fácil encontrar una conducta creativa en los estudiantes, algunas caracte- 
rísticas que pueden favorecer su desarrollo como, por ejemplo, el hecho de prestar más atención al proceso que al resultado, ser disciplinado y dispuesto al trabajo duro, ser autocrítico, se encuentran más a menudo en las mujeres que en los hombres (Maslow, 1983).

\section{GÉNERO, MATEMÁTICAS Y TECNOLOGÍA}

En los últimos veinte años ha habido también interés en estudiar las posibles implicaciones que puede tener, para las diferencias de género, el uso de la tecnología como apoyo para la enseñanza y el aprendizaje de las matemáticas. Desde finales de los años ochenta, Apple (1989) expresaba su preocupación indicando que, a pesar de los esfuerzos de los expertos para que esto no ocurra, los planes de estudios de matemáticas y ciencias contribuyen con mucha frecuencia a la reproducción de las diferencias de género. Consideraba que esta situación podría empeorar con la incorporación de la tecnología como apoyo para el aprendizaje. En un estudio llevado a cabo en Inglaterra, Hoyles y Sutherland (1989) reportaron no haber encontrado diferencias de género significativas en cuanto a motivación, persistencia, iniciativa y ansiedad al usar la computadora en un ambiente Logo en el cual se propiciaba el trabajo en parejas y se dejaba a los alumnos en libertad de planear y desarrollar sus propios proyectos. Sin embargo, al comparar entre sí parejas integradas sólo por niños o sólo por niñas encontraron algunas diferencias en la actitud hacia el trabajo, la manera de trabajar, la naturaleza de la colaboración, así como en el tipo de discusión que se generaba. También detectaron diferencias en la manera cómo los niños y las niñas usaban las computadoras para desarrollar sus proyectos y el tipo de proyectos que planeaban. Si bien observaron que en ambos había una actitud de colaboración, notaron que los varones tendían a competir entre ellos y difícilmente cedían ante los argumentos del compañero, mientras las niñas tendían mucho más hacia un trabajo de cooperación.

Considerando que el uso de las computadoras para enseñar matemáticas es cada vez más común, Forgasz (2002) investigó cuáles son las creencias de los estudiantes acerca de este uso de la computadora. Como parte de este mismo estudio recabó datos acerca de las actitudes de los estudiantes hacia las matemáticas, las computadoras y las matemáticas enseñadas con computadora. Los resultados mostraron que, si bien, la mayoría ya no considera a las matemáticas como un dominio masculino, sí consideran que los varones son más competentes que las mujeres en el uso de la tecnología. Pero, con respecto al uso de las computadoras para enseñar matemáticas, sus respuestas fueron más ambivalentes.

Si bien muy interesantes, estos resultados no son generalizables, dado que se pueden deber a características particulares, propias de cierto tipo de educación y cultura. O, como señala Yelland (2001), en muchas ocasiones las supuestas diferencias de género se pueden deber al contexto y a la manera de plantear la actividad. Por lo tanto, en los estudios que investigan las diferencias de género, es fundamental tomar en cuenta, por un lado, el ambiente sociocultural particular en el cual se trabaja; $y$, por el otro, es conveniente considerar que existen múltiples maneras de incorporar la tecnología en el salón de clase y que los efectos pueden depender fuertemente del ambiente particular que se va creando.

\section{EMAT: UN PROYECTO MEXICANO}

Desde 1997 la Secretaría de Educación Pública en México promueve un proyecto nacional, llamado EMAT (Enseñanza de las Matemáticas con Tecnología), en el que la tecnología (computadoras y calculadoras TI-92) se usa para apoyar la enseñanza de las matemáticas en la escuela secundaria (estudiantes entre 12 y 15 años de edad). Al momento de realizar el estudio que aquí reportamos, se estaban usando en el proyecto distintos paquetes computacionales para apoyar la enseñanza de distintos temas de matemáticas: la hoja electrónica de cálculo para la enseñanza de la aritmética, el preálgebra y el álgebra; Cabri Géomètre para la enseñanza de la geometría; SimCalc MathWorlds para acercar a los alumnos a la idea de variación y sus diferentes representaciones; y Stella para modelar matemáticamente situaciones simples.

Para la ejecución del proyecto se siguieron dos vertientes: el diseño de actividades a desarrollar con los alumnos en el salón de clase; y la formación de los profesores participantes en el proyecto. Las actividades se presentaban en hojas de trabajo y, en su mayoría, trataban temas que aparecen en el currículo mexicano de matemáticas para el nivel medio básico. En las hojas de trabajo se presentaba un problema, en ocasiones se recordaba algún conocimiento previo que se esperaba que los alumnos ya poseyeran y, a través de indicaciones o preguntas, se guiaba la actividad del alumno. El uso de este tipo de hojas de trabajo representaba una manera novedosa de trabajar en la clase, tanto para los alumnos como para los profesores. Para desarrollar la actividad que se presentaba en la hoja de trabajo se pedía siempre a los alumnos usar alguno de los paquetes computacionales ya mencionados. En la figura 1 se presenta una hoja de trabajo como ejemplo.

En cuanto a la formación de profesores se pueden distinguir esencialmente dos aspectos importantes: por un lado, se impartieron una serie de talleres en los cuales se proporcionó instrucción para que los profesores pudieran manejar los paquetes computacionales y la calculadora TI-92; por el otro, y ésta fue la parte más novedosa y relevante de la formación de los profesores, se discutieron las implicaciones didácticas y pedagógicas de la propuesta de uso de la tecnología que propone EMAT (Ursini y Rojano, 2000). En particular, una característica fundamental del acercamiento que se propone en EMAT consiste en ir de la práctica y los ejemplos particulares a los principios teóricos generales, y no al revés. Este acercamiento difiere de manera sustancial del que comúnmente se usa en nuestras escuelas donde, por lo general, la parte teórica precede a la práctica y a los ejercicios. 
Figura 1

Ejemplo de hoja de trabajo.

Nombre:

ÁlgEBRA Y NUEVAS IDEAS

Edad:

Fecha:

En 1990 vivían en nuestro país aproximadamente 80 millones de habitantes. Si consideramos que el territorio mexicano tiene una extensión de casi dos millones de kilómetros cuadrados, ¿cuántas personas crees que había en promedio por cada kilómetro cuadrado? Al número de habitantes por kilómetro cuadrado se le llama densidad de población.

En esta actividad conocerás y aplicarás un método para calcular el crecimiento de la población mexicana y cómo éste se refleja en su densidad de población.

Para empezar, construye una hoja de cálculo de acuerdo con las siguientes instrucciones:

1) En la columna A escribe 1990 y encuentra una fórmula para generar una serie que aumente de diez en diez cada año.

2) En la columna B escribe la cantidad de habitantes que había en México en 1990. Para calcular las poblaciones subsecuentes, establece un porcentaje de crecimiento, digamos $25 \%$ (esto se puede precisar consultando los resultados del censo más reciente). Enseguida, escribe en la columna B3 la fórmula $=\mathrm{B} 2+0,25 * \mathrm{~B} 2$ (la población anterior más $25 \%$ ) y cópiala más abajo.

3) En las columnas C2 escribe la fórmula = B 2 / 2.000.000 (población/superficie), que calcula la densidad poblacional respectiva y cópiala más abajo.

\begin{tabular}{|c|c|c|c|c|}
\hline & A & B & C & D \\
\hline 1 & Año & Población & $\begin{array}{c}\text { Densidad } \\
\text { Habitante por } \mathrm{Km}^{2}\end{array}$ & \\
\hline 2 & 1990 & 80.000 .000 & 40 & \\
\hline 3 & 2000 & 100.000 .000 & 50 & \\
\hline 4 & 2010 & 125.000 .000 & 63 & \\
\hline
\end{tabular}

¿Qué densidad habrá en el año 2100?

¿En qué año la densidad llegará a 10.000 habitantes por $\mathrm{km}^{2}$ ?

Discute estos resultados y sus implicaciones con tus compañeros.

En los talleres para los profesores se puso, además, un énfasis especial en resaltar la dinámica a seguir en el salón de clase. Se recomendó acomodar las computadoras en forma de herradura para facilitar el trabajo de los estudiantes en parejas o tercias, y para que el profesor pudiera observar el trabajo que estaban realizando. Sólo después de una breve introducción al tema, el profesor les entregaría la hoja de trabajo correspondiente y los invitaría a desarrollar la actividad propuesta trabajando en equipo. Cabe mencionar que ésta no es la dinámica que se sigue usualmente en el salón de clase, donde, por lo general, los estudiantes conforman una audiencia pasiva, que escucha las explicaciones del profesor y resuelve los ejercicios propuestos de forma individual.

En los talleres, se recalcó también que, al trabajar en EMAT, el papel del profesor era radicalmente distinto al que esta- ban acostumbrados en sus clases sin tecnología, donde solían dictar cátedra, mantener la atención de los alumnos hacia el pizarrón y hacerlos trabajar de manera individual. Ahora su función sería la de promover el trabajo en equipo, observar con cuidado el trabajo que desarrollan las parejas o tercias al seguir las instrucciones y preguntas planteadas en las hojas de trabajo, contestar las preguntas o dudas de los alumnos, hacer sugerencias, proponer acercamientos y organizar discusiones de grupo de manera periódica para llegar a un consenso acerca de los conceptos matemáticos involucrados en las actividades. En síntesis, participar en el proyecto implicaba, en cierta forma, la introducción en el aula de una cultura diferente, cambiando las normas y la práctica de la enseñanza de las matemáticas.

Una vez concluidos los talleres para la formación de los docentes, los participantes recibieron las hojas de trabajo con 
las actividades que desarrollarían los estudiantes. La primera versión de las hojas de trabajo empleadas en el proyecto EMAT se pueden consultar en Mochón, Rojano y Ursini (2000a) para la parte de aritmética, preálgebra y álgebra; en Zubieta, Martínez, Rojano y Ursini (2000) para la parte de geometría; y en Mochón, Rojano y Ursini (2000b) para variación y modelación. Se recomendó a los profesores que las analizaran y resolvieran ellos mismos antes de llevarlas a sus alumnos. Cabe señalar que al inicio del proyecto EMAT la gran mayoría de los profesores no tenía experiencia en el uso de la computadora o la calculadora TI-92, ni de cómo usarlas para apoyar la clase de matemáticas.

El proyecto se inició con 16 profesores y 667 estudiantes. Al término del tercer año, cuando se realizó el estudio que reportamos en este artículo, trabajaban en EMAT 89 profesores y aproximadamente 10.000 estudiantes.

\section{PROPÓSITO DEL ESTUDIO}

Como ya se mencionó en la introducción, algunas de las cuestiones que nos interesa responder son las siguientes:

- ¿Usar la tecnología en la clase de matemáticas repercute en el comportamiento de los alumnos y las alumnas en el aula de matemáticas?

- ¿Los cambios de comportamiento que se observan son iguales para los hombres y para las mujeres?

- ¿Los cambios de comportamiento contribuyen a que las diferencias de género se acentúen o a que haya una mayor equidad de género?

Para ello se decidió indagar, en un primer momento, cómo perciben los profesores los eventuales cambios de conducta de sus estudiantes durante la clase de matemáticas y analizar si, desde la perspectiva de los docentes, existen diferencias de género en los comportamientos observados.

La mayoría de los aspectos que decidimos investigar ya habían sido estudiados, resaltando las diferencias de género, por otros investigadores en otros países. Sin embargo, salvo algunas excepciones (Hoyles y Sutherland, 1989; Yelland, 2001), estos estudios se habían realizado en aulas de matemáticas sin tecnología. Los aspectos acerca de los cuales se decidió recabar información fueron los siguientes:

1) Participación (comenta las tareas propuestas con el maestro o compañeros; interviene en las discusiones de grupo).

2) Capacidad para analizar un problema (entiende el problema propuesto; puede analizar los resultados obtenidos en la pantalla de la computadora o de la calculadora para contestar las preguntas de las hojas de trabajo).

3) Capacidad para interpretar correctamente las hojas de trabajo (puede seguir las indicaciones que aparecen en las hojas de trabajo y entiende el propósito de las preguntas).
4) Iniciativa (propone posibles soluciones a los problemas planteados sin consultarlo con el profesor; puede tomar decisiones de manera autónoma).

5) Solicitud de ayuda (pide ayuda al profesor o a un compañero para desarrollar la tarea propuesta).

6) Dedicación al trabajo (se involucra en la tarea y persiste en ella).

7) Defensa de sus ideas (puede sostener sus puntos de vista con el maestro y compañeros).

8) Creatividad (resuelve los problemas propuestos de una manera original y, en ocasiones, desarrolla actividades no indicadas explícitamente en las hojas de trabajo).

9) Preferencia por el trabajo en equipo o por el trabajo individual (prefiere trabajar en pares/ternas y coopera en la solución de la tarea o prefiere trabajar solo/sola de manera independiente).

Estos aspectos serían estudiados considerando al alumno como sujeto de la comunidad que integra el aula de matemáticas, sin tomar en cuenta su vida y experiencia fuera de la misma.

\section{METODOLOGÍA}

En este estudio participaron, de manera voluntaria, 24 profesores (15 hombres y 9 mujeres). Sus edades estaban entre los 25 y los 58 años $(\mu=41,5)$; su antigüedad como profesores de matemáticas iba de 5 a 31 años $(\mu=17)$; y su experiencia en haber trabajado en el proyecto EMAT era de un año (1 profesor), dos años (16 profesores) y tres años (6 profesores). Antes de que se iniciara el proyecto EMAT, estos profesores, igual que la mayoría de sus colegas, acostumbraban a dictar cátedra y solicitaban a sus alumnos que, de preferencia, trabajaran de forma individual, como ellos mismos lo fueron comentando a lo largo del presente estudio.

Al empezar el estudio se discutieron los nueve aspectos arriba mencionados con los 24 docentes, con el propósito de lograr una comprensión compartida del significado de cada uno de estos aspectos. Se solicitó posteriormente a cada profesor que escogiera, según su disposición, uno, dos o tres grupos de alumnos que estaban participando en el proyecto EMAT y que calificara el comportamiento de cada uno de esos alumnos. Para ello se les pidió usar la siguiente escala:

1: cuando el aspecto considerado no se había presentado nunca;

2: cuando se había presentado con poca frecuencia;

3: cuando se había presentado con mucha frecuencia.

El aspecto 9 (preferencia por el trabajo en equipo o por el trabajo individual) se calificaría con 3 cuando el alumno/alumna mostrara preferencia para trabajar en pares/ternas, 2 si no mostraba una preferencia específica y 1 si prefería trabajar de manera individual. 
Para obtener información de cómo iba cambiando la conducta de los alumnos en la clase de matemáticas, en relación con el tiempo que habían estado usando la tecnología en las clases de matemáticas, se pidió a los profesores que calificaran estudiantes que habían estado trabajando uno, dos y tres años en el proyecto EMAT.

Siguiendo estas indicaciones, los docentes calificaron la conducta de un total de 1.113 estudiantes (568 hombres y 545 mujeres) cuyas edades oscilaban entre los 12 y los 15 años. La gran mayoría de éstos provenía de clase media baja y pertenecían a siete escuelas ubicadas en tres ciudades de la República Mexicana. Estaban cursando $1^{\circ}$, $2^{\circ}$ o $3^{\circ}$ año de secundaria y su experiencia en el proyecto era de uno, dos o tres años, distribuidos de la siguiente manera: 576 habían trabajado en EMAT durante un año; 459 durante dos años; y 78 estudiantes tenían tres años en EMAT. El número de estos últimos es reducido, dado que se trataba de estudiantes con los cuales se había iniciado el proyecto en esas escuelas. En la tabla I se muestra su distribución por sexo, grado escolar y tiempo en el proyecto. Todos estos estudiantes habían trabajado con la calculadoras TI-92; adicionalmente, 597 de ellos habían estado trabajando con la Hoja Electrónica de Cálculo, y los otros 516 con Cabri Géomètre.

Tabla I

Distribución de las frecuencias absolutas de los alumnos por grado y por años en EMAT.

\begin{tabular}{|c|c|c|c|c|c|c|}
\hline \multirow{2}{*}{} & \multicolumn{5}{|c|}{$\begin{array}{c}\text { Años en } \\
\text { EMAT }\end{array}$} \\
\cline { 2 - 7 } & \multicolumn{2}{|c|}{$\mathbf{1}$} & \multicolumn{2}{c|}{$\mathbf{2}$} & \multicolumn{2}{c|}{} \\
\hline $\begin{array}{c}\text { Grado } \\
\text { escolar }\end{array}$ & Alumnos & Alumnas & Alumnos & Alumnas & Alumnos & Alumnas \\
\hline $1^{\circ}$ & 276 & 239 & 0 & 0 & 0 & 0 \\
\hline $2^{\circ}$ & 14 & 27 & 162 & 161 & 0 & 0 \\
\hline $3^{\circ}$ & 13 & 7 & 66 & 70 & 27 & 51 \\
\hline
\end{tabular}

Las calificaciones que los profesores asignaron a los estudiantes, en los nueve aspectos evaluados, constituyeron un primer grupo de datos que fue analizado mediante pruebas estadísticas de significación. El objetivo era detectar si dichas calificaciones sugerían cambios de comportamiento en los estudiantes considerando dos condiciones generales:

- estudiantes del mismo sexo con uno, dos y tres años en EMAT;

- estudiantes de sexo distinto con uno, dos o tres años en EMAT.

En el primer caso obtendríamos información acerca de los posibles cambios de conducta percibidos por los profesores entre alumnos del mismo sexo pero con distinto tiempo de estar usando la tecnología en la clase de matemáticas. Los resultados del segundo análisis permitirían detectar si las calificaciones otorgadas por los profesores reflejaban diferencias de género entre alumnos y alumnas con el mismo tiempo en el proyecto.

Las pruebas estadísticas empleadas fueron la de KruskalWallis y la $\chi^{2}$. Se optó por la prueba de Kruskal-Wallis por ser un método alternativo a un análisis de varianza, pero para datos ordinales. Es útil para examinar la significatividad de la diferencia entre grupos de conjuntos de calificaciones o puntajes provenientes de varios grupos o muestras que se toman simultáneamente, además requiere que las muestras sean independientes. En este estudio, la condición de independencia se cumplía, los datos eran ordinales y estaban agrupados por el sexo de los estudiantes (masculino o femenino) y por su tiempo en el proyecto EMAT (uno, dos o tres años). La prueba de Kruskal-Wallis nos permitió examinar si existían diferencias significativas en las calificaciones que los profesores asignaron a los estudiantes en los nueve aspectos evaluados, tomando en consideración el sexo y su tiempo en EMAT.

En cuanto a la prueba $\chi^{2}$, también es un procedimiento estadístico no paramétrico, para datos que puedan ser categorizados o tratados en un nivel nominal. Permite comparar la frecuencia observada entre dos categorías, y evalúa si contienen o no frecuencias equivalentes. Esencialmente, el dato que se emplea para su cálculo es la frecuencia en que ocurre cada categoría estudiada. La hipótesis implícita es que todas las categorías tienen la misma frecuencia. En el caso de nuestro estudio, se contabilizó el número de veces que los profesores asignaban a los estudiantes la categoría «nunca», «con poca frecuencia» $\mathrm{y}$ «con mucha frecuencia», en los nueve aspectos observados. La comparación de frecuencias se hizo para todos los aspectos observados, según el sexo de los estudiantes y sus años en EMAT. Las calificaciones dadas por los profesores se tomaron como datos nominales. Con la prueba $\chi^{2}$ se evaluaría la significatividad de las diferencias encontradas.

Los resultados estadísticos fueron interpretados de manera independiente por los cuatro investigadores participantes en este estudio y discutidos conjuntamente de modo que fuera posible hacer una comparación de perspectivas.

Con el propósito de profundizar acerca de los resultados obtenidos a partir del análisis estadístico de los datos, se procedió a conformar un segundo bloque de datos recabando información de los profesores mediante una entrevista. Se entrevistaron cuatro de los profesores participantes en el estudio: un profesor y una profesora con dos años de experiencia en EMAT, y un profesor y una profesora con tres años. Se trató de una entrevista semiestructurada, en la cual se les preguntó si las conclusiones a las que apuntaban los resultados globales obtenidos iban de acuerdo con su experiencia personal; y si podían, a partir de su experiencia personal, explicar estos resultados. Esto último, con el propósito de conocer los argumentos que usaban los profesores para explicar las diferencias de conducta observadas entre alumnos del mismo sexo pero con distinto tiempo en el proyecto, y entre alumnos de sexo distinto con el mismo tiempo en el proyecto. Estas explicaciones revelarían los eventuales estereotipos de género de los profesores. 


\section{RESULTADOS Y DISCUSIÓN}

\section{Comparación entre estudiantes del mismo sexo con uno, dos y tres años en el proyecto}

En este primer análisis se compararon las calificaciones otorgadas por los profesores a estudiantes del mismo sexo pero con distinto tiempo de trabajar en el proyecto. El propósito era ver si los profesores, a través de las calificaciones otorgadas, manifestaban haber percibido cambios de conducta entre alumnos del mismo sexo pero con distinto tiempo de estar usando la tecnología en la clase de matemáticas. En la tabla II se presenta, para cada categoría y para cada sexo, la distribución del porcentaje de estudiantes por años en EMAT, que fueron calificados con: 1 = nunca, 2 = con poca frecuencia, $3=$ con mucha frecuencia.

Para cada categoría se aplicó la prueba $\chi^{2}$, lo que permite saber si hubieron diferencias significativas entre estos porcentajes. La variable principal en este primer análisis es el tiempo de permanencia en el proyecto.
Los resultados indican que, para la mayoría de los aspectos observados, existen diferencias significativas entre estudiantes del mismo sexo, cuando los datos se agrupan de acuerdo a los años que tienen en el proyecto. Se observa también que, para algunos aspectos, la permanencia en el proyecto parece incidir de manera similar en los hombres y en las mujeres, pero en otros aspectos incide de manera distinta.

Los datos muestran que los profesores consideran que hay diferencias significativas en el comportamiento de los estudiantes varones con uno, dos o tres años en el proyecto en los siguientes aspectos:

- capacidad para analizar el problema que se les plantea y los resultados que se obtienen en pantalla de la computadora o la calculadora (aspecto 2);

- capacidad para interpretar las hojas de trabajo (aspecto 3);

- iniciativa que muestran al enfrentarse a un problema (aspecto 4);

Tabla II

Distribución de los porcentaje por sexo comparando años en EMAT.

Nota: $* \mathrm{p}<0.01 \quad * * \quad \mathrm{p}<0.05$

\begin{tabular}{|c|c|c|c|c|c|c|c|c|c|}
\hline \multirow{3}{*}{ Aspecto } & \multirow{3}{*}{ Calificación } & \multirow{2}{*}{\multicolumn{3}{|c|}{$\begin{array}{c}\text { Hombres } \\
\text { Años en EMAT }\end{array}$}} & \multirow{3}{*}{$\chi^{2}$} & & Mujeres & & \multirow{3}{*}{$\chi^{2}$} \\
\hline & & & & & & & en EM & & \\
\hline & & $1(\mathrm{n}=303)$ & $2(n=228)$ & $\begin{array}{c}3(\mathrm{n}= \\
27)\end{array}$ & & $\begin{array}{c}1(\mathrm{n}= \\
273)\end{array}$ & $\begin{array}{c}2(\mathrm{n}= \\
231)\end{array}$ & $\begin{array}{c}3(\mathrm{n}= \\
51)\end{array}$ & \\
\hline \multirow{3}{*}{ Participación } & 1 & 25,8 & 29,8 & 18,5 & \multirow{3}{*}{5} & 10,4 & 21,7 & 11,8 & \multirow{3}{*}{$25.37 *$} \\
\hline & 2 & 41,1 & 41,9 & 33,3 & & 46,4 & 40,2 & 21,6 & \\
\hline & 3 & 32,2 & 28,4 & 48,1 & & 42,8 & 37,7 & 66,7 & \\
\hline \multirow{3}{*}{$\begin{array}{l}\text { Capacidad } \\
\text { de análisis }\end{array}$} & 1 & 25,5 & 27,9 & 0 & \multirow{3}{*}{$19 *$} & 14,8 & 18,9 & 7,8 & \multirow{3}{*}{$16.25^{*}$} \\
\hline & 2 & 48,8 & 47,9 & 40,7 & & 57,6 & 48 & 39,2 & \\
\hline & 3 & 25,2 & 24,2 & 59,3 & & 27,2 & 32,8 & 52,9 & \\
\hline \multirow{3}{*}{ Interpretación } & 1 & 21,8 & 32,6 & 0 & \multirow{3}{*}{$28,43 *$} & 15,2 & 22,5 & 9,8 & \multirow{3}{*}{$25,41^{*}$} \\
\hline & 2 & 50,9 & 42,8 & 37 & & 55,2 & 45,1 & 29,4 & \\
\hline & 3 & 26,7 & 24,7 & 63 & & 29,2 & 32 & 60,8 & \\
\hline \multirow{3}{*}{ Iniciativa } & 1 & 28,8 & 43,3 & 18,5 & \multirow{3}{*}{$17,48^{*}$} & 15,6 & 37,3 & 25,5 & \multirow{3}{*}{$48,23 *$} \\
\hline & 2 & 43,3 & 37,2 & 40,7 & & 56,4 & 33,2 & 25,5 & \\
\hline & 3 & 27,3 & 19,1 & 40,7 & & 27,6 & 29,1 & 49 & \\
\hline \multirow{3}{*}{ Solicitud de ayuda } & 1 & 19,6 & 23,3 & 33,3 & \multirow{3}{*}{8,58} & 10 & 25,8 & 11,8 & \multirow{3}{*}{$48,23 * *$} \\
\hline & 2 & 55,2 & 57,2 & 63 & & 65,2 & 47,1 & 37,3 & \\
\hline & 3 & 24,5 & 19,5 & 3,7 & & 24 & 26,6 & 51 & \\
\hline \multirow{3}{*}{ Dedicación } & 1 & 16,6 & 24,7 & 0 & \multirow{3}{*}{$15,18^{*}$} & 11,2 & 17,2 & 2 & \multirow{3}{*}{$48,23 *$} \\
\hline & 2 & 50 & 45,1 & 44,4 & & 50 & 42,6 & 9,8 & \\
\hline & 3 & 32,5 & 30,2 & 55,6 & & 38,8 & 39,8 & 88,2 & \\
\hline \multirow{3}{*}{ Defensa de ideas } & 1 & 31,9 & 50,7 & 33,3 & \multirow{3}{*}{$21,61 *$} & 28,8 & 45,1 & 15,7 & \multirow{3}{*}{$48,23 *$} \\
\hline & 2 & 43,9 & 32,6 & 33,3 & & 53,6 & 33,6 & 23,5 & \\
\hline & 3 & 23,6 & 16,3 & 33,3 & & 17,2 & 20,1 & 60,8 & \\
\hline \multirow{3}{*}{ Creatividad } & 1 & 27,9 & 44,2 & 25,9 & \multirow{3}{*}{$23,93 *$} & 21,6 & 32,4 & 29,4 & \multirow{3}{*}{$25,38^{*}$} \\
\hline & 2 & 49,4 & 38,6 & 29,6 & & 58,8 & 42,2 & 29,4 & \\
\hline & 3 & 22,1 & 17,2 & 44,4 & & 19,2 & 24,6 & 41,2 & \\
\hline & 1 & 16 & 26,5 & 18,5 & & 12 & 16,8 & 7,8 & \\
\hline Trabajo en equipo & 2 & 49,1 & 30,7 & 37 & $20,8^{*}$ & 41,6 & 40,6 & 33,3 & 6,79 \\
\hline & 3 & 34 & 42,8 & 44,4 & & 46 & 42,2 & 58,8 & \\
\hline
\end{tabular}


- dedicación al trabajo (aspecto 6);

- defensa de sus ideas (aspecto 7);

- creatividad en la solución de problemas y en la forma de enfrentar una tarea (aspecto 8);

- preferencia por el trabajo en equipo o por el trabajo individual (aspecto 9).

En todos estos aspectos los varones con 3 años en el proyecto obtienen las calificaciones más altas, lo que sugiere un impacto positivo del trabajo en EMAT.

No se encuentran cambios significativos entre los estudiantes varones con uno, dos o tres años en el proyecto en:

- participación (aspecto 1);

- solicitud de ayuda (aspecto 5).

En relación con las alumnas, los datos muestran que los profesores consideran que hay diferencias significativas en el comportamiento de las alumnas con uno, dos o tres años en el proyecto en los siguientes aspectos:

- participación (aspecto 1);

- capacidad para analizar el problema que se les plantea y los resultados que obtienen en pantalla de la computadora o la calculadora (aspecto 2);

- capacidad para interpretar las hojas de trabajo (aspecto 3);

- iniciativa que muestran al enfrentarse a un problema (aspecto 4);

- solicitud de ayuda (aspecto 5);

- dedicación al trabajo (aspecto 6);

- defensa de sus ideas (aspecto 7);

- creatividad en la solución de problemas y en la forma de enfrentar una tarea (aspecto 8).

No se encuentran cambios significativos entre las estudiantes mujeres con uno, dos o tres años en el proyecto en:

- su preferencia por el trabajo en equipo o por el trabajo individual (aspecto 9).

Según las calificaciones otorgadas por los profesores, la mayoría de los estudiantes con tres años en el proyecto, e independientemente de su sexo, habían desarrollado una buena capacidad para interpretar las hojas de trabajo (aspecto 3); además podían analizar los problemas que se les planteaban, e interpretaban los resultados que obtenían (aspecto 2) mejor que sus compañeros con menos tiempo en EMAT.

Recordemos que, al iniciar su trabajo en el proyecto, los estudiantes, por lo general, no estaban acostumbrados a emplear hojas de trabajo y tenían cierta dificultad para leerlas y comprenderlas. Según nuestra experiencia, al inicio de su trabajo en el proyecto, la mayoría de los estudiantes suelen esperar que el profesor lea las hojas de trabajo, explique su contenido e indique explícitamente en qué consiste el problema a resolver. Pero, como lo indican los resultados, la gran mayoría de los estudiantes no tiene mayores dificultades para adaptarse a la nueva modalidad de trabajo y, según los profesores, logran asumir la responsabilidad de leer e interpretar las hojas, así como de analizar por ellos mismos los resultados que obtienen en la pantalla de la computadora o de la calculadora. Esto fue corroborado durante las entrevistas. Los profesores atribuyeron la mejoría de los alumnos a la permanencia en el proyecto y a los elementos didácticos que en él participan. $\mathrm{Al}$ respecto, una maestra comentó: «Se van desarrollando más habilidades, se han acostumbrado a hacer el trabajo solos sin necesitar tanto nuestra ayuda, lo he notado en el salón. Ya se acostumbraron a que llegue primero un problema, las explicaciones ellos mismos se las dan, pasan al pizarrón y les explican a los compañeros.» Otra maestra comentó en el mismo sentido: «Los alumnos me dicen que está muy fácil. Muchos de los que en el bimestre pasado sacaron 5 (calificación reprobatoria) están bien entrados en el trabajo. Yo lo atribuyo al apoyo de la calculadora.»

Lo anterior resulta notable sobre todo al analizar las calificaciones que los profesores otorgaron a los varones. En el caso de las mujeres, los cambios no fueron tan llamativos. Resulta que aún después de tres años en EMAT había, según los profesores, alumnas que no podían interpretar correctamente las hojas de trabajo o los resultados que obtenían en la pantalla de la computadora. Al preguntar a una maestra si consideraba que las niñas tenían dificultades para comprender los problemas e interpretar los resultados, comentó: «Yo creo que sí. Las niñas no encuentran siempre las respuestas solas.»

Los varones con tres años en el proyecto manifestaban también bastante más iniciativa (aspecto 4) en la manera de resolver los problemas planteados que sus pares con menos tiempo en el proyecto. Además, disminuyó notablemente el número de los que nunca mostraban algún tipo de iniciativa. Si bien un mayor porcentaje de mujeres con tres años en EMAT recibieron calificaciones más altas que sus compañeras con menos tiempo en el proyecto, llama la atención que un porcentaje bastante alto no muestra tener iniciativa alguna. De hecho, este porcentaje es notablemente mayor que el correspondiente a alumnas con un solo año en el proyecto. No tenemos comentarios de los profesores al respecto, pero consideramos que, si bien se trata sólo de una minoría, sería importante investigar más a fondo acerca de las causas que parecen inhibir el desarrollo de esta capacidad en ciertas niñas cuando usan la tecnología, llevándolas más bien a seguir instrucciones y no a desarrollar acercamientos que implican una mayor iniciativa.

Tanto las alumnas como los alumnos, con tres años en EMAT, manifestaban más dedicación (aspecto 6) que sus compañeras y compañeros con menos tiempo en el proyecto. Un profesor comentó: «Gracias a EMAT empiezan a trabajar hasta aquéllos que son flojos.»

En cuanto a la capacidad para defender sus propias ideas (aspecto 7), las alumnas con tres años en el proyecto sostenían y defendían sus puntos de vista mucho mejor que sus compañeras con menos tiempo en el proyecto. A este respecto una maestra comentó: «En un principio 
son pocas las alumnas que toman decisiones, cuando pasa el tiempo, dada la misma retroalimentación que les da la máquina, aumentan su confianza y llegan hasta a debatir.» Para los varones también encontramos que el porcentaje de los que defendían bien sus puntos de vista era más alto cuando se trataba de alumnos con tres años en el proyecto. Estos datos sugieren que el uso de la tecnología en la clase de matemáticas ayudó a ambos, hombres y mujeres, a mejorar y desarrollar la capacidad para defender sus puntos de vista, pero, en el caso de los hombres, este cambio de conducta fue menos notable. En referencia a esta situación, una profesora comentó: «En el salón tradicional saben que están bien sólo si los maestros se lo decimos, con EMAT ellos mismos validan si están bien o no.»

Según Maslow (1982), la creatividad (aspecto 8) es una característica difícil de encontrar en los estudiantes; sin embargo, los profesores involucrados en el proyecto consideraron que éste tuvo un influjo positivo en la creatividad. Al ser entrevistada, una profesora explicó: «Creo que los estudiantes desarrollan su creatividad cuando buscan una posible solución al problema al que se enfrentan. Aunque siguen las instrucciones de las hojas, cuando se dan cuenta de que algo está mal buscan otra aproximación. Esto es evidente en especial cuando trabajan con las computadoras usando Cabri.» Otro profesor comentó: «La creatividad de los alumnos se desarrolla mucho. Desarrollan diferentes estrategias, diferentes maneras de resolver un problema y encuentran soluciones generales, aprenden cómo generalizar.» Los datos de la tabla II indican que los profesores consideran que los varones y las mujeres con tres años en el proyecto manifestaban más creatividad que sus compañeros con uno o dos años de trabajar en él. Sin embargo, el porcentaje de los estudiantes que nunca demostraban una actitud creativa es también alto. Estos datos sugieren que, cuando se trata de un estudiante creativo, sea hombre o mujer, el trabajo con la computadora ayuda a que su creatividad se manifieste y se incremente; pero, cuando se trata de un estudiante con poca creatividad, la computadora no propicia necesariamente que su creatividad se desarrolle.

En los demás aspectos (1, 5 y 9), los años de trabajo en el proyecto parecen incidir de manera algo distinta en hombres y mujeres. Por ejemplo, en participación (aspecto 1) y en solicitud de ayuda (aspecto 5) no se encontraron diferencias significativas entre los varones con distinto tiempo en el proyecto, pero sí entre las mujeres. Las alumnas con tres años en el proyecto participaban más que sus compañeras con menos experiencia y solicitaban ayuda con más frecuencia que sus compañeras con menos tiempo en el proyecto. De allí se colige que la experiencia del trabajo con la computadora contribuye a que las alumnas se involucren más activamente en el trabajo, participando y solicitando ayuda. Las diferencias observadas entre las chicas con más y menos tiempo en el proyecto confirman lo anterior y nos permiten concluir que la combinación del uso de la herramienta con el modelo pedagógico propuesto por EMAT propicia una actitud más activa en las mujeres. Este resultado refuerza lo encontrado por Boaler (1996) que señala que un enfoque didáctico más abierto ayuda a que las chicas se involucren más.

En lo que se refiere a los varones, los profesores entrevistados manifestaron que, si bien los alumnos que llevaban tres años en el proyecto mostraban una actitud más positiva hacia el trabajo, esto no se reflejaba en un cambio sustancial en su participación en el trabajo de grupo. De acuerdo con los profesores, esta diferencia entre los y las estudiantes que llevaban tres años en el proyecto obedece a cuestiones de género: «Las mujeres tienen mucha seguridad en sus argumentos y debates, ya no son las mujeres tímidas que no hablan, su autoestima se fortalece, la retroalimentación que ofrece la computadora hace que, independientemente de lo que el maestro diga, defiendan sus puntos de vista.»

En cuanto a la preferencia por el trabajo en equipo o por el trabajo individual (aspecto 9), no se encontró entre las mujeres una diferencia significativa en correspondencia con los años de experiencia en usar la tecnología. En cambio, trabajar con la tecnología aumentó la preferencia por el trabajo en equipo entre los varones, si bien ésta resultó ser siempre menor que la que manifestaron las mujeres. Un maestro comentó: «Los varones al principio trabajaban muy egoístamente, ahora ya se ayudan más entre ellos. Otro de mis problemas era lograr que trabajaran con las niñas. Ahora sí hay una colaboración bonita.» Respecto a las niñas comentó: «Las niñas son más fáciles de adaptarse.» Otra maestra declaró lo siguiente: «Las niñas trabajando en equipo se sienten más seguras, es más reconfortante para ellas, les da más confianza.»

Un análisis general de estos resultados permite ver cómo los estudiantes varones no parecen involucrarse demasiado en actividades que implican la interacción con otros compañeros o compañeras, ni con el profesor. Esto es lo que indican los resultados que se refieren a la participación, la solicitud de ayuda y el trabajo en equipo. La excepción la encontramos en la defensa de sus propias ideas, pero esta conducta, más que implicar cierta interacción con otro en un sentido colaborativo, conlleva conductas de confrontación, debate y argumentación con las cuales los estudiantes varones parecen sentirse más cómodos.

Otro resultado que llama la atención es que los profesores asignaron las calificaciones más bajas a los estudiantes que tenían dos años en el proyecto. Esto sucede para todos los aspectos observados e independientemente del sexo de los estudiantes. Interrogados al respecto, los profesores coincidieron en que este resultado no se podía atribuir al trabajo con la tecnología. Comentaron que la falta de atención e interés era una característica de los estudiantes de esa edad y era igual para ambos sexos. Hay que señalar que entre los estudiantes con dos años en el proyecto había unos cuantos que cursaban el tercer año de secundaria; pero este no era el caso de la mayoría. Éstos cursaban el segundo año de secundaria y su edad oscilaba entre los 13 y los 14 años. Los profesores manifestaron que la falta de atención e interés no se manifestaba exclusivamente en la clase de matemáticas, se había observado en otras asignaturas y solía presentarse recurrentemente con alumnos de segundo de secundaria. Según ellos, para explicarlo era necesario considerar otros 
factores, por ejemplo, el desarrollo físico y emocional propio de la edad, que conlleva inquietudes e intereses extraescolares que los vuelven más inquietos y distraídos. Una profesora comentó: «Pienso que estos resultados no dependen del uso de la tecnología [...] en esta edad los estudiantes no están interesados en lo que nosotros les enseñamos; no comprenden su utilidad. En general, cuando los estudiantes comienzan la escuela secundaria son muy entusiastas, durante el segundo año su interés baja dramáticamente, pero en el tercer año comienzan a entender la utilidad de lo que están aprendiendo y su interés nuevamente crece. Esto no depende de EMAT, se debe a su propio desarrollo.»

\section{Comparación entre estudiantes de sexo distinto con uno, dos o tres años en el proyecto}

El propósito de este segundo análisis es detectar si las calificaciones otorgadas por los profesores reflejan diferencias de género entre los alumnos y las alumnas con el mismo tiempo en el proyecto. En la tabla III se presentan nuevamente los datos que se habían incluido en la tabla II, pero organizados con el propósito de facilitar la comparación entre los y las estudiantes con distinto tiempo en EMAT. La variable principal en este análisis es el sexo de los alumnos.

Tabla III

Distribución de los porcentajes de cada opción respuesta por años en EMAT y sexo de los estudiantes.

\begin{tabular}{|c|c|c|c|c|c|c|c|}
\hline \multirow{3}{*}{ Aspecto } & \multirow{3}{*}{ Calificación } & \multicolumn{6}{|c|}{ Años en EMAT } \\
\hline & & \multicolumn{2}{|c|}{1} & \multicolumn{2}{|c|}{2} & \multicolumn{2}{|c|}{3} \\
\hline & & $\begin{array}{l}\text { Alumnos } \\
(\mathrm{n}=303)\end{array}$ & $\begin{array}{l}\text { Alumnas } \\
(\mathrm{n}=273)\end{array}$ & $\begin{array}{l}\text { Alumnos } \\
(\mathrm{n}=228)\end{array}$ & $\begin{array}{l}\text { Alumnas } \\
(\mathrm{n}=231)\end{array}$ & $\begin{array}{c}\text { Alumnos } \\
(\mathrm{n}=27)\end{array}$ & $\begin{array}{l}\text { Alumnas } \\
(\mathrm{n}=51)\end{array}$ \\
\hline \multirow{3}{*}{$\begin{array}{l}\text { Participación } \\
a^{*}, b^{* *}\end{array}$} & 1 & 25,8 & 10,4 & 29,8 & 21,7 & 18,5 & 11,8 \\
\hline & 2 & 41,1 & 46,4 & 41,9 & 40,2 & 33,3 & 21,6 \\
\hline & 3 & 32,2 & 42,8 & 28,4 & 37,7 & 48,1 & 66,7 \\
\hline \multirow{3}{*}{$\begin{array}{l}\text { Capacidad } \\
\text { de análisis } \\
a^{*}, b^{*}\end{array}$} & 1 & 25,5 & 14,8 & 27,9 & 18,9 & 0 & 7,8 \\
\hline & 2 & 48,8 & 57,6 & 47,9 & 48 & 40,7 & 39,2 \\
\hline & 3 & 25,2 & 27,2 & 24,2 & 32,8 & 59,3 & 52,9 \\
\hline \multirow{3}{*}{$\begin{array}{l}\text { Interpretación } \\
b^{*}\end{array}$} & 1 & 21,8 & 15,2 & 32,6 & 22,5 & 0 & 9,8 \\
\hline & 2 & 50,9 & 55,2 & 42,8 & 45,1 & 37 & 29,4 \\
\hline & 3 & 26,7 & 29,2 & 24,7 & 32 & 63 & 60,8 \\
\hline \multirow{3}{*}{$\begin{array}{l}\text { Iniciativa } \\
a^{*}\end{array}$} & 1 & 28,8 & 15,6 & 43,3 & 37,3 & 18,5 & 25,5 \\
\hline & 2 & 43,3 & 56.4 & 37,2 & 33,2 & 40,7 & 25,5 \\
\hline & 3 & 27,3 & 27,6 & 19,1 & 29,1 & 40,7 & 49 \\
\hline \multirow{3}{*}{$\begin{array}{l}\text { Solicitud de ayuda } \\
a^{*}, b^{* *}, c^{*}\end{array}$} & 1 & 19,6 & 10 & 23,3 & 25,8 & 33,3 & 11,8 \\
\hline & 2 & 55,2 & 65,2 & 57,2 & 47,1 & 63 & 37,3 \\
\hline & 3 & 24,5 & 24 & 19,5 & 26,6 & 3,7 & 51 \\
\hline \multirow{3}{*}{$\begin{array}{l}\text { Dedicación } \\
c^{*}\end{array}$} & 1 & 16,6 & 11,2 & 24,7 & 17,2 & 0 & 2 \\
\hline & 2 & 50 & 50 & 45,1 & 42,6 & 44,4 & 9,8 \\
\hline & 3 & 32,5 & 38,8 & 30,2 & 39,8 & 55,6 & 88,2 \\
\hline \multirow{3}{*}{$\begin{array}{l}\text { Defensa de ideas } \\
a^{* *}, b^{* *}, c^{* *}\end{array}$} & 1 & 31,9 & 28,8 & 50,7 & 45,1 & 33,3 & 15,7 \\
\hline & 2 & 43,9 & 53,6 & 32,6 & 33,6 & 33,3 & 23,5 \\
\hline & 3 & 23,6 & 17,2 & 16,3 & 20,1 & 33,3 & 60,8 \\
\hline \multirow{3}{*}{$\begin{array}{l}\text { Creatividad } \\
b^{* *}\end{array}$} & 1 & 27,9 & 21,6 & 44,2 & 32,4 & 25,9 & 29,4 \\
\hline & 2 & 49,4 & 58,8 & 38,6 & 42,2 & 29,6 & 29,4 \\
\hline & 3 & 22,1 & 19,2 & 17,2 & 24,6 & 44,4 & 41,2 \\
\hline \multirow{3}{*}{$\begin{array}{l}\text { Trabajo en equipo o } \\
\text { individual } \\
a^{* *}, b^{* *}\end{array}$} & 1 & 16 & 12 & 26,5 & 16,8 & 18,5 & 7,8 \\
\hline & 2 & 49,1 & 41,6 & 30,7 & 40,6 & 37 & 33,3 \\
\hline & 3 & 34 & 46 & 42,8 & 42,2 & 44,4 & 58,8 \\
\hline
\end{tabular}

Nota: Se aplicaron pruebas $\chi^{2}$ para evaluar las diferencias en las frecuencias obtenidas, entre alumnos y alumnas, por tiempo en EMAT. En la primera columna de la tabla se indica, con las letras en cursiva, en qué año hubo diferencias significativas: $a$ en primer año; $b$ en segundo; y $c$ en tercer año en EMAT. Además: * $\mathrm{p}<.01, * * \mathrm{p}<.05$ 
En la tabla III se puede observar que, para la mayoría de las categorías e independientemente del tiempo en el proyecto, las alumnas recibieron calificaciones más altas que los alumnos. Se nota también que hay más diferencias significativas entre los y las estudiantes con uno o dos años de haber empezado a usar la tecnología, que entre aquéllos y aquéllas que tenían tres años de experiencia en EMAT.

En seis aspectos se encontraron diferencias significativas entre alumnos y alumnas con un año en el proyecto: participación, capacidad de análisis, iniciativa, solicitud de ayuda, defensa de ideas y preferencia por trabajo individual o en equipo. Entre los chicos y las chicas con dos años en EMAT hubo diferencias significativas en siete aspectos: participación, capacidad de análisis, interpretación, solicitud de ayuda, defensa de ideas, creatividad y preferencia por trabajo individual o en equipo. Y, entre los que llevaban tres años en el proyecto, las diferencias fueron significativas sólo en tres aspectos: solicitud de ayuda, dedicación y defensa de ideas. Una hipótesis que se desprende de lo anterior es que el uso de la tecnología en la clase de matemáticas propicia que disminuyan las diferencias en el comportamiento que manifiestan hombres y mujeres. Tal suposición se requiere comprobar con estudios ulteriores, dado que, al momento de realizar este estudio, el número de estudiantes con tres años en el proyecto era aún pequeño en comparación con los que llevaban en el proyecto uno o dos años.

Sin embargo, hay que, señalar que, al formular esta hipótesis, no nos estamos basando sólo en las calificaciones otorgadas por los profesores, también estamos tomando en cuenta los comentarios que hicieron los cuatro profesores durante las entrevistas.

A continuación se presenta el análisis de los datos obtenidos para cada uno de los nueve aspectos, junto con los comentarios de los profesores entrevistados. Durante la entrevista, los profesores manifestaron estar de acuerdo con los resultados obtenidos del análisis estadístico de los datos y consideraron que éstos reflejaban su propia experiencia con los alumnos. Además, dieron argumentos para explicar y justificar esos resultados. Es interesante notar que dichos argumentos difieren entre sí de manera notable, a veces parecen hasta contradecirse, $\mathrm{y}$ reflejan claramente sus propios estereotipos de género.

\section{Participación}

Los datos muestran que, según los profesores, las alumnas suelen participar en la clase de matemáticas más que los alumnos. Lo anterior parece ser independiente del tiempo que lleven trabajando con la tecnología. Al respecto, una profesora opinó: «Los hombres no participan, son conformistas, se conforman con lo que dicen los demás. Las niñas participan, escuchan las ideas de sus compañeros, a veces las refutan y entre ellas mismas refutan las ideas (sic) que dan a una pregunta. Muchos hombres son tan pasivos, que no hacen nada y se conforman con lo que dicen otros.» Otro profesor comentó: «Usualmente las alumnas discuten las tareas propuestas entre ellas mismas y con el profesor mucho más que los niños [...] las niñas intervienen más durante la discusión en grupo.» Esta apreciación, común entre los profesores, coincide con lo reportado por otros investigadores cuando señalan que la participación de las niñas en la clase de matemáticas puede llegar a rebasar la de los niños (Subirats y Brullet, 1999).

Los datos de la tabla III indican diferencias significativas entre los dos sexos para estudiantes con uno o dos años en el proyecto. Esta diferencia es en ambos casos a favor de las mujeres. Sin embargo, en los estudiantes con tres años de experiencia, esta diferencia ya no resulta significativa, si bien la distribución de porcentajes sigue favoreciendo a las mujeres. Este dato resulta interesante porque sugiere que al trabajar con la tecnología también los varones empiezan a participar más en la clase de matemáticas. Hay que señalar, sin embargo, que a pesar de la tecnología sigue habiendo un porcentaje relativamente alto de hombres y mujeres que participan poco o nunca.

\section{Capacidad para analizar un problema}

También en este aspecto se encontraron diferencias significativas entre los estudiantes con uno o dos años en EMAT. En ambos casos los profesores consideraron que las alumnas tienen mejor capacidad para analizar los problemas que se presentan en las hojas de trabajo y los resultados que obtienen en la pantalla de la computadora o de la calculadora. Estas diferencias entre hombres y mujeres desaparecen cuando se consideran estudiantes con tres años en el proyecto. En general, la capacidad de análisis de los estudiantes, independientemente de su sexo, es muy notable después de tres años en el proyecto.

\section{Capacidad para interpretar correctamente las ho- jas de trabajo}

En cuanto a la interpretación de las hojas de trabajo, se encuentra una diferencia significativa sólo entre los alumnos y las alumnas con dos años en EMAT. En este caso las mujeres parecen desempeñarse mejor que los varones. Al respecto, una maestra que estaba atendiendo estudiantes en su $2^{\circ}$ año en el proyecto comentó: «Las niñas interpretan mejor los resultados. Ellas realizan, resuelven y preguntan. Si se le hacen preguntas directas contestan. Los hombres contestan menos.» Por lo contrario, no se encuentran diferencias significativas entre estudiantes con uno y tres años en EMAT. Sin embargo, se observa una buena capacidad para interpretar las hojas de trabajo, en ambos sexos a los tres años de participar en el proyecto.

\section{Iniciativa}

En este aspecto se encontraron diferencias significativas entre los chicos y las chicas con uno o dos años en el proyecto. Al respecto, un maestro comentó: «Las niñas tienen más iniciativa que los niños al principio del trabajo con EMAT, pero los hombres poco a poco 
se están emparejando. Las mujeres toman más iniciativas, hacen más cosas por ellas mismas. Pero siempre preguntan antes de dar la respuesta para ver si está bien como lo están haciendo.» Otra maestra aclaró: «Se ha visto que las mujeres tienen mayor iniciativa, no sólo en matemáticas, en todas las materias.» Pero, como lo señalaba el maestro, esta diferencia entre mujeres y hombres ya no se encuentra en los que han trabajado ya tres años con la tecnología. La diferencia entre ellos ya no es significativa y los porcentajes indican que ambos sexos muestran un alto grado de iniciativa, en particular los varones. También llama la atención que, si bien el porcentaje correspondiente a las mujeres con mucha iniciativa supera al de los hombres, el porcentaje de ellas que muestra muy poca iniciativa es también más alto que el de los hombres. Esto sugiere que el trabajo con la tecnología favorece más a los hombres que a las mujeres en cuanto a desarrollar la capacidad para tomar iniciativas. Hay un porcentaje relativamente alto de mujeres que, al trabajar con la tecnología, no logran desarrollar comportamientos que implican cierta dosis de iniciativa. Las razones para ello pueden ser múltiples y encontrarlas ameritaría un estudio más profundo en esta dirección.

\section{Solicitud de ayuda}

Otro resultado interesante se refiere a la solicitud de ayuda. Para este aspecto, la diferencia entre los dos sexos es significativa siempre. Según los profesores, en general y no sólo al trabajar en el proyecto, son siempre las mujeres las que solicitan más ayuda. Este resultado difiere de lo reportado por estudios como el realizado por Subirats y Brullet (1999), donde se señala que, por lo general, son los varones quienes solicitan más atención y ayuda por parte de los maestros. Un maestro comentó al respecto: «Las niñas piden siempre más ayuda que los niños, tanto de aspectos técnicos como de matemáticas. Los niños preguntan menos, ya que, si alguno lo hace, los demás se burlan de él.» Una maestra comentó: «Si su cabeza [refiriéndose a las niñas] dice no puedo es no puedo. Aunque en realidad sí puedan, están esperando que venga alguien a ayudarles y los niños no, ellos le buscan.» $\mathrm{Al}$ analizar estos y otros comentarios que los profesores hicieron en cuanto a la solicitud de ayuda, saltan a la vista las diferentes maneras que tienen de interpretar esta conducta. En ocasiones estas interpretaciones resultan hasta contradictorias entre sí, revelando diferentes perspectivas de género. Por ejemplo, algunos profesores consideraron que, al pedir ayuda, las niñas manifestaban su deseo de conocer más, de asegurarse que las respuestas que brindaban eran correctas, su deseo por perfeccionar el trabajo que realizaban. Otros, por lo contrario, interpretaron esta conducta por parte de las alumnas como pasividad, dependencia del profesor y una tendencia a aparentar incapacidad. En cuanto a los alumnos varones, algunos profesores interpretaron su tendencia de no solicitar ayuda como independencia y autonomía. Otros interpretaron esa misma conducta como egoísmo, al no querer compartir su trabajo, dificultades de comunicación y expresión, falta de concentración e interés, y temor a la burla.

\section{Dedicación al trabajo}

Otras investigaciones (Figueiras et al., 1998) han señalado que, por lo general, las niñas son más dedicadas y constantes en su trabajo. Los datos obtenidos con este estudio confirman sólo parcialmente esta tendencia, ya que, según los profesores, tanto los varones como las mujeres mostraban dedicación al trabajo en clase. Si bien las mujeres obtuvieron puntuaciones un poco más altas, no se encontraron diferencias significativas entre ambos sexos con uno o dos años en el proyecto. Sin embargo, aparecen diferencias significativas, a favor de las mujeres, entre estudiantes con tres años en EMAT. Durante la entrevista, un profesor comentó: «Las niñas son más dedicadas que los niños, las niñas se concentran en su hoja de trabajo y tratan de resolverla. Los niños no prestan mucha atención a la hoja de trabajo, se concentran en otras cosas, exploran más los demás recursos que ofrece la computadora. Las niñas tienden a seguir instrucciones, los niños se sienten más libres para explorar.» Otra maestra declaró: «Niños y niñas son dedicados, pero los niños lo son menos que las niñas [...] no son tan persistentes, sienten que no tienen que trabajar tanto, se sienten culturalmente superiores (sic).» En estos comentarios se pone de manifiesto cierta postura de género. Por ejemplo, en el primer comentario, si bien el profesor reconoce que las niñas son más dedicadas, atribuye esta conducta a su pasividad, ya que, como afirma, «las niñas tienden a seguir instrucciones». Por otro lado, considera que la falta de dedicación de los niños se debe a su afán por explorar qué otros recursos ofrece la computadora. En el segundo comentario vemos que la profesora atribuye la menor dedicación de los varones al hecho de que éstos se sienten, según ella, «superiores» por el hecho de ser varones y esto los lleva a considerar «que no tienen que trabajar tanto» como las mujeres.

\section{Defensa de sus ideas}

Para este aspecto se encontró que las mujeres siempre reciben calificaciones significativamente más altas que los varones, independientemente del tiempo que llevan en EMAT. Una profesora hizo el siguiente comentario: «Los niños son muy pasivos, aceptan el punto de vista de los demás muy fácilmente. Las niñas escuchan los argumentos de los demás pero, cuando no están de acuerdo, argumentan también. Cuando están convencidas de alguna idea discuten en el equipo[...] Los niños son obstinados, creen que nunca se equivocan, son testarudos; las niñas reflexionan y, cuando están convencidas, defienden sus ideas.» Sin embargo, los profesores observan una ligera mejoría en este aspecto también en los varones. Una profesora comentó: «Sí, por lo general las niñas defienden mejor sus puntos de vista, pero también los niños van mejorando. Un niño el otro día me decía "Yo estoy bien, maestra, fíjese". Y yo revisé a la ligera diciéndole que estaba mal, pero él insistió en que me fijara bien en lo que había hecho. Y en efecto, tenía razón él, estaba bien. Me dijo "Ya ve, con mi método, pero estoy bien, tenía razón”. Esto no se daba antes.» Una vez más se observa cómo en estas declaraciones se van reflejando las posturas de género de los profesores, por ejemplo, cuando 
afirman, generalizando, que «los niños son muy pasivos [...] son obstinados, creen que nunca se equivocan, son testarudos» y que «las niñas escuchan los argumentos de los demás[...] reflexionan, defienden sus ideas».

\section{Creatividad}

Los porcentajes reportados en la tabla III indican que se encontraron diferencias significativas para este aspecto sólo entre los alumnos y las alumnas con dos años en el proyecto. Según los profesores, las mujeres estaban manifestando más creatividad en su manera de abordar las tareas que los hombres. No se encontraron diferencias significativas al considerar los estudiantes con uno y tres años en el proyecto. Los resultados muestran que los profesores consideran que los estudiantes que llevan tres años en EMAT, hombres o mujeres, manifiestan bastante creatividad. Este dato confirma lo encontrado por Morales y sus colegas (1998), quienes señalan que el trabajo con la computadora promueve el desarrollo de la creatividad independientemente del sexo del alumno. Cuestionado al respecto, un profesor comentó: «Sí, hay más creatividad que antes. Por ejemplo, unos alumnos hicieron con Cabrí un cubo en forma tridimensional de colores y ellos solos empezaron a modificar área, perímetro y volumen. La creatividad se modifica bastante. Ellos solos aprendieron a ocultar las líneas, y me hicieron sudar para entender su trabajo tridimensional. Fue iniciativa de una niña el de ocultar los trazos.» Una profesora señaló: «La creatividad se desarrolla mucho con EMAT. Encuentran otras estrategias, otros caminos para resolver la tarea y llegan más pronto a generalizaciones.» Otra profesora dio un ejemplo: «Estábamos viendo ángulos dentro de la circunferencia, además habíamos visto los elementos de la circunferencia, qué cuerda, qué tangente, etc. A muchos niños ya les habían dado esa explicación en los talleres, ahora la finalidad del taller es que apoyen las asignaturas. Los hombres del taller de moldeo ya la traían por escrito y se conformaron con la definición que traían, que era de diccionario o del libro. Las niñas en cambio sacaron sus propios conceptos como ellas lo entendían y se notaba la diferencia de las definiciones, pero llegaban a lo mismo. Bueno, no todas las niñas son así, también hay niñas poco creativas.» Si bien todos los profesores entrevistados coincidieron en señalar que, al trabajar en el proyecto, tanto los chicos como las chicas habían desarrollado su creatividad, hubo quien apreciaba más el trabajo de las chicas («Las niñas en cambio sacaron sus propios conceptos como ellas lo entendían y se notaba la diferencia de las definiciones [...]») que el trabajo de los chicos («Los hombres del taller de moldeo ya la traían por escrito y se conformaron con la definición que traían, que era de diccionario o del libro.»), reflejando así su percepción de las diferencias de género.

\section{Preferencia por el trabajo en equipo o por el tra- bajo individual}

Para este aspecto se encontraron diferencias significativas entre los y las estudiantes con uno y dos años en el proyecto siendo siempre las mujeres las que privilegian el trabajo en equipo. Al respecto una maestra comentó:
«Los niños son más individualistas, si están solos están mejor. De repente me dicen: "Maestra, esa computadora está sola, déjeme pasarme para allá”. En cambio las niñas me dicen: "Maestra déjeme pasar con esa niña".» Otra profesora confirmó: «Las niñas prefieren trabajar en equipo, se sienten más seguras, para ellas es más reconfortante, les da más confianza. Los hombres tienden a ser más solitarios.» Este resultado confirma lo encontrado por Hoyles y Sutherland (1989), quienes observaron que, si bien al trabajar con Logo en ambos sexos había cooperación, los varones tendían mucho más hacia el trabajo competitivo que las mujeres; mientras ellas tendían más hacia la cooperación. Sin embargo, los datos reportados en la tabla III muestran que ya no aparecen diferencias significativas entre alumnos y alumnas con tres años en el proyecto. A este cambio se refiere el siguiente comentario: "Así era al principio, a estas alturas ya no. Hay una interacción bonita entre ambos sexos y entre los niños también. Ahora hasta los muy buenos trabajan con otros menos buenos. Al principio de año no era así. Yo promuevo mucho el trabajo en equipo.» Este resultado sugiere que la permanencia en el proyecto llevó a reducir un poco las diferencias entre hombres y mujeres en lo que se refiere a este aspecto, propiciando así una mayor equidad de género.

\section{CONCLUSIONES}

El propósito de este estudio era investigar si cambia y cómo cambia el comportamiento de los alumnos y las alumnas en la clase de matemáticas cuando se usa la tecnología como apoyo didáctico. En particular, nuestro objetivo era indagar si cuando se usa la tecnología procurando crear un ambiente que invita a la discusión, al trabajo en equipo y al intercambio de ideas, se propicia una mayor equidad o se refuerzan las diferencias de género. Un primer paso para empezar a dar respuesta a nuestras preguntas consistió en recurrir a algunos profesores que usaban la tecnología como soporte para enseñar matemáticas e indagar cómo ellos percibían los cambios de conducta de sus alumnos y de sus alumnas en el aula.

Un primer análisis de los datos obtenidos mostró que la introducción de la tecnología en la clase de matemáticas, junto con la propuesta pedagógica que promueve el proyecto EMAT, implica una modificación de la cultura en el salón de clases que lleva a cambios significativos en el comportamiento de los y las estudiantes. Según los profesores, después de tres años en el proyecto, la gran mayoría de los estudiantes, sin distinción de sexo, tenían una buena capacidad para analizar los problemas que se les planteaban y para interpretar las hojas de trabajo, mostraban tener más iniciativa que sus compañeros con menos tiempo en el proyecto, eran más dedicados al trabajo, defendían mejor sus ideas y tenían una actitud más creativa al enfrentarse a los problemas que se les planteaban. De acuerdo con los profesores hubo también aspectos para los cuales los cambios observados fueron muy distintos para los alumnos y las alumnas. Por ejemplo, los varones con tres años en el proyecto mostraban tener más preferencia por el trabajo en equipo 
que sus compañeros con uno o dos años en el proyecto, si bien un porcentaje bastante elevado seguía prefiriendo el trabajo individual. Por el contrario, no reportaron cambios significativos en este aspecto entre las alumnas, que preferían, en su gran mayoría, el trabajo en equipo. Tampoco reportaron cambios significativos entre los varones en participación y solicitud de ayuda, mientras sí los señalaron para las mujeres. Según los profesores, las mujeres con tres años en el proyecto participaban más y solicitaban más ayuda que sus compañeras que habían usado la tecnología menos tiempo. Estos resultados muestran que cierto uso de la tecnología en la clase de matemáticas propicia cambios de conducta importantes entre los estudiantes en general y que aquéllos no son iguales en los hombres y las mujeres.

Un segundo análisis de los datos, en el que se compararon las calificaciones obtenidas por estudiantes de sexo distinto con uno, dos o tres años en el proyecto, muestra que las diferencias entre los comportamientos que manifiestan en la clase de matemáticas los alumnos y las alumnas con tres años en el proyecto, tienden a ser mucho menores que las detectadas entre aquéllos con uno o dos años. Este resultado sugiere que usar la tecnología en un ambiente en el que se propicia el trabajo en equipo, las discusiones de grupo y en el que se guía el trabajo de los alumnos a través de hojas de trabajo puede ayudar a que se vayan modificando ciertos patrones culturales de conducta que contribuyen a reforzar las diferencias de género $\mathrm{y}$, de este modo, se puede ayudar a lograr una mayor equidad.

Algunos de los aspectos conductuales observados en el estudio, como, por ejemplo, solicitud de ayuda en el salón de clase, al parecer están muy influidos por patrones culturales externos al aula. En un estudio futuro sería importante considerar referentes culturales, en población mexicana, externos al salón de clase, para conocer cómo se reflejan dentro del aula.

Con el fin de complementar los resultados obtenidos en este estudio, actualmente se está desarrollando una investigación acerca de las actitudes hacia las matemáticas y hacia las matemáticas enseñadas con el apoyo de la tecnología que tienen los estudiantes que están participando en el proyecto EMAT.

En el presente estudio se pudo observar también que los profesores calificaron las conductas de sus estudiantes como positivas o negativas, de acuerdo con sus propios estereotipos de género, y atribuyeron estas conductas a características de personalidad, como si éstas fueran inherentes a uno u otro sexo. Esto podría influir en su forma de interactuar con los chicos y las chicas en la clase de matemáticas, estereotipando previamente a los estudiantes e interactuando con ellos, en relación con sus propias concepciones de género. Consideramos conveniente realizar, en un futuro próximo, un estudio longitudinal con el propósito de investigar si los estereotipos de género que manifiestan los profesores sufren alguna modificación a consecuencia de los cambios que presentan los estudiantes cuando trabajan en un ambiente con tecnología, abierto, del tipo que promueve el proyecto EMAT. Queremos señalar también que se encontraron diferencias entre los profesores y las profesoras en su manera de calificar a sus estudiantes. En general, las profesoras otorgaban calificaciones más bajas. No se profundizó en este aspecto, pero sería importante desarrollar investigaciones en esta dirección.

Finalmente, queremos recordar que este estudio se realizó con estudiantes de un nivel socioeconómico medio bajo. Para investigaciones futuras sería interesante dirigirse a estudiantes de diferentes estratos socioeconómicos y analizar cómo influyen éstos en los cambios de conducta que se observaron en el presente estudio.

\section{AGRADECIMIENTO}

Los autores agradecen a la Dra. Ana Isabel Sacristán sus valiosos comentarios y sugerencias, que ayudaron a mejorar la presentación de este artículo.

\section{NOTA}

Esta investigación estuvo patrocinada por el Consejo Nacional de Ciencia y Tecnología, Proyecto de Grupo G26338S. 


\section{REFERENCIAS BIBLIOGRÁFICAS}

APPLE, M.W. (1989). Maestros y textos: Una economía política de las relaciones de clase y de sexo en educación. Buenos Aires: Paidós.

BEN-CHAIN, D., LAPPAND, G. y HOUANG, R. T. (1985) Visualizing rectangular solids made of small cubes: Analizing and effecting students' performance. Educational Studies in Mathematics, 16, pp. 389-409.

BOALER, J. (1996). Respuestas por géneros a enfoques matemáticos abiertos y cerrados. 20 años de investigación cooperativa en género y matemáticas -dónde estamos, hacia dónde vamos. Sesiones de IOWME. Grupo de trabajo 6: Género y Matemáticas. ICME 8, p. 101. Sevilla,

COOPER, C.R., MARQUIS, A. y AYERS-LÓPEZ, S. (1982). Peer learning in the classroom: Training developmental problems and consequences of children's spontaneous interactions. Wilkinson. Communicating in the Classroom.

FENNEMA, E. y SHERMAN, J.A. (1976). Fennema-Sherman Attitude Scales. Catalog of selected documents in psychology, 6, p. 31 .

FIGUEIRAS, L., MOLERO M., SALVADOR, A. y ZUASTI, N. (1998). Género y matemáticas. Madrid: Síntesis.

FORGASZ, H.J. (2001). Australian and US preservice teachers' perceptions of the gender stereotyping of mathematics. Van den Heuvel-Panhuizen, M. (ed.). Proceedings of the $25^{\text {th }}$ Conference of the International Group for the Psychology of Mathematics Education, pp. 2-433 - 2-440. Utrecht University, Holanda.

FORGASZ, H.J. (2002). Computers for Learning Mathematics: Gendered Beliefs, en Cockburn A.D. y Nardi E. (eds.). Proceedings of the $26^{\text {th }}$ Conference of the International Group for the Psychology of Mathematics Education, pp. 2-368 - 2-375. Norwich. Gran Bretaña.

FORGASZ, H.J. y LEDER, G.C. (2000). The «mathematics as a gendered domain» scale, en Nakahara, T. y Koyama, M. (eds.). Proceedings of the $24^{\text {th }}$ Conference of the International Group for the Psychology of Mathematics Education, pp. 2273 - 2-279. Hiroshima University, Japón.

HANNA, G. (1989). Mathematics achievement of girls and boys in grade eight: Results from twenty countries. Educational Studies in Mathematics, 20, pp. 225-232.

HOYLES, C. y SUTHERLAND, R. (1989). Logo Mathematics in the Classroom, Londres y Nueva York: Routledge.

HELWIG R., ANDERSON L. y TINDAL G. (2001). Influence of Elementary Student Gender on Teachers' Perceptions of Mathematics Achievement. The Journal of Educational Research, 95(2), pp. 93-102.

KLOOSTERMAN, P. (1990). Attributions, performance following failure and motivation in mathematics. Fennema, E. y Leder, G.C. (eds.). Mathematics and gender, pp. 96127. Nueva York: Teachers College Press.

KOEHLER, M. S. (1990). Classrooms, Teachers, and gender differences in mathematics, en Fennema, E. y Leder, G.C. (eds.). Mathematics and gender, pp.128-148. Nueva York: Teachers College Press.
KUYPER, H. y VAN DER WERF, M.P.C. (1990). Math teachers and gender differences in math achievement: Math participation and attitudes towards math, en Booker, G., Cob, P. y Mendicuti, T. (eds.). Proceedings of the fourteenth Conference of the International Group for the Psychology of Mathematics Education, pp. 143-150. México.

LEDER, G.C. (1992) Mathematics and Gender: Changing perspectives, en Grows, D. A. (ed.). Handbook of Research on Mathematics Teaching and Learning, pp. 597-622. EE.UU.: National Council of Teachers of Mathematics.

LEDER, G.C. (1996). Equity in the mathematics classroom: beyond the rhetoric, en Parker, L. H. (ed.). Gender, Science and Mathematics, pp. 95-104. Holanda: Kluwer Academic Publishers.

LEDER, G.C. (2001). Mathematics as a gender domain: New measurement tools. Annual Meeting of American Education Research Association (AERA). Seatle, EEUU.

LEDER, G.C. y FENNEMA, E. (1990). Gender differences in mathematics a synthesis, en Fennema, E. y Leder, G. C. (eds.). Mathematics and gender, pp.188-200. Nueva York: Teachers College Press.

MASLOW, A. (1982). La personalidad creadora. Barcelona: Kairós.

MEYER, M.R. y KOEHLER, M.S. (1990). Internal Influences on Gender Differences in Mathematics, en Fennema E. y Leder, G.C. (eds.). Mathematics and Gender, pp. 60-95. Nueva York y Londres: Teachers College, Columbia University.

MIDDLETON, J. A. y SPANIAS, P. A. (1999). Motivation for achievement in mathematics: Findings generalizations and criticism of the research. Journal for Research in Mathematics Education, 30, pp. 65-88.

MOCHÓN, S., ROJANO, T. y URSINI, S. (2000a). «Matemáticas con la hoja electrónica de cálculo». Dirección General de Materiales y Métodos Educativos. SEP, ILCE, CONACYT. México.

MOCHÓN, S., ROJANO, T. y URSINI, S. (2000b). «Modelación y matemáticas del cambio». Dirección General de Materiales y Métodos Educativos. SEP, ILCE, CONACYT. México.

MORALES, C., TURCOTT, V., CAMPOS, A. y LIGNAN, L. (1998). Actitudes de los escolares hacia la computadora y los medios para el aprendizaje. Tecnología y comunicaciones educativas. ILCE. México.

SUBIRATS, M. y BRULLET, C. (1999). Rosa y azul, en Belausteguigoitia, M. y Mingo, A. (eds.). Géneros prófugos: feminismo y educación, pp. 189-223. México: Paidós.

URSINI, S. y ROJANO, T. (2000). Guía para integrar los talleres de capacitación EMAT. SEP-ILCE. México.

YELLAND, N. (2001). Girls, Mathematics and Technology, en Atweh, B., Forgasz, H. y Nebres, B. (eds.). Sociocultural Research on Mathematics Education. An International Perspective. LEA Publishers.

ZUBIETA, G., MARTÍNEZ, A., ROJANO, T. y URSINI, S. (2000). «Geometría dinámica». Dirección General de Materiales y Métodos Educativos. SEP, ILCE, CONACYT. México. 